\title{
APROPIACIÓN TECNOLÓGICA CON PIZARRÓN INTERACTIVO Y TABLETAS DIGITALES EN PROFESORES DE EDUCACIÓN BÁSICA
}

\author{
TECHNOLOGICAL APPROPRIATION WITH INTERACTIVE \\ WHITEBOARD AND DIGITAL TABLETS IN BASIC EDUCATION \\ TEACHERS
}

Judith Verónica Escobar; jvescobar@yahoo.com

Instituto Godwin, México

Leonardo David Glasserman; glasserman@gmail.com

Centro Regional de Formación Docente e Investigación Educativa, México

María Soledad Ramírez; solramirez@tecvirtual.mx

Tecnológico de Monterrey, México

\section{RESUMEN}

Los procesos de apropiación tecnológica de docentes de educación primaria se presentan como un tema interesante para integrar estrategias mediadas por tecnologías en el aula. La investigación partió de la interrogante ¿Cómo se da la apropiación tecnológica de docentes de educación primaria de primer y tercer grado, que utilizan estrategias de enseñanza vinculadas a dispositivos tecnológicos como el pizarrón interactivo y tabletas digitales para el reforzamiento de conceptos matemáticos? Se siguió una metodología cualitativa con enfoque de estudio de casos; empleando como técnicas de recolección de datos entrevistas, análisis de documentos significativos, ejercicios y formatos de observación, donde la validez de la información se aseguró mediante la triangulación de instrumentos. Los resultados obtenidos indicaron hallazgos en los niveles de apropiación de acceso y adopción al usar pizarrón interactivo y tabletas digitales. También se detectó 
que no se desarrolló el nivel de apropiación tecnológica, donde el factor tiempo de uso pudo tener una influencia en el resultado.

PALABRAS CLAVE: Apropiación tecnológica, tabletas digitales, pizarrón interactivo.

\section{ABSTRACT}

The processes of technological appropriation of primary teachers are presented as interesting strategies for integrating technology in the classroom. The research question was as follows: How technological appropriation of $1^{\text {st }}$. and $3^{\text {rd }}$ primary teachers is developed by using teaching strategies related to technological devices such as the interactive whiteboard and digital tablets to reinforce math concepts? A qualitative methodology was followed with a case study approach; collection techniques included interview data, analysis of significant documents, exercises and observation formats, where the validity of the information is assured by triangulation of instruments. The results showed findings in the levels of access and adoption in the use of interactive whiteboard and digital tablets. Also, it was found that the level of technological appropriation was not developed, where the time factor could have had an influence on the results.

KEYWORDS: Technological Appropriation, digital tablets, interactive whiteboard.

\section{INTRODUCCIÓN}

La incorporación de las Tecnologías de la Información y la Comunicación (TIC) dentro del sector educativo provoca retos a la labor docente, por su rol de guía y ejemplo, para que evolucione a la par de los recursos digitales y tecnológicos y promueva, en conjunto con éstos, estrategias de enseñanza más dinámicas e interactivas, acordes con las nuevas generaciones de estudiantes. Por ello, hoy en día un gran número de instituciones educativas buscan desarrollar estrategias que permitan a los docentes cambiar de un rol tradicionalista como proveedor de la información, hacia acciones más activas al ser facilitador de la información (Glasserman, 2013).

El cambio de rol tradicionalista en el sistema de enseñanza implica, entre otras cosas, un cambio de actitud de los profesores ante la tecnología y una motivación para desarrollar una apropiación tecnológica de apoyo a su docencia, misma que se relacionará con los cambios que se produzcan en ellos, como consecuencia del empleo de la tecnología en su quehacer educativo, a través de diversas etapas de apropiación (Celaya, Lozano y Ramírez, 2010; Cobo, 2005; Ramírez, 2009). En la educación básica se están integrando tecnologías que tienen un impacto en las estrategias que utilizan los docentes.

En este entorno, surgió el interés del estudio que aquí se presenta para indagar ¿Cómo se da la apropiación tecnológica de docentes de educación primaria de primer y tercer grado, 
que utilizan estrategias de enseñanza vinculadas a dispositivos tecnológicos como el pizarrón interactivo y tabletas digitales para el reforzamiento de conceptos matemáticos?

\section{MARCO CONCEPTUAL}

Actualmente, los docentes requieren contar con un perfil que les permita hacer frente a las nuevas demandas educativas del siglo XXI. En este sentido, González (2008) menciona que es fundamental que el profesor adquiera ciertas habilidades, conocimientos y actitudes que lo capaciten para aplicar estrategias innovadoras y modelos alternos que incluyan la enseñanza por medio de las tecnologías de información y comunicación (TIC), las cuales se apoyan de modalidades de aprendizaje que tienen características diferentes en cuanto a dispositivos tecnológicos y recursos digitales utilizados, de tal forma que el alumno tenga un rol activo y mayor responsabilidad de su aprendizaje en el proceso (Gómez y Alemán, 2011).

El proceso de apropiación tecnológica dará como resultado llevar la teoría educativa en la práctica y en la aplicación de resultados para potenciar y enriquecer los procesos de enseñanza y aprendizaje estimulando la creatividad, la colaboración, el conocimiento adaptable y la formación continua, entre otras destrezas (Guzman y Nussbaum, 2009). Esta apropiación se dará en tres niveles: acceso, adopción y apropiación y su evaluación será la forma en que los usuarios evalúan y adoptan, adaptan e integran la tecnología en sus prácticas cotidianas (Carroll, Howard, Peck y Murphy, 2003).

Las prácticas mencionadas contemplan la utilización de estrategias de enseñanza que deben ser seleccionadas y aplicadas de acuerdo con el contexto de la clase, con las características de los estudiantes, con el contenido curricular, con la intención o meta, con la mejora del proceso de enseñanza-aprendizaje y con el contexto creado por los alumnos (Díaz-Barriga y Hernández, 2002).

Muy particularmente, para la asignatura de matemáticas se argumenta que la tecnología informática tiene el potencial para la creación de un entorno propicio y una buena atmósfera para las actividades de matemáticas, promoviendo la creatividad de la enseñanza y nuevas formas de aprender (Mistretta, 2005; Yushau, Mji y Wessels, 2005). Otros autores apuntan a que existe una gran variedad de hardware, software, herramientas basadas en la web, recursos libres de Internet, aprendizaje basado en juegos que están disponibles para la enseñanza de las matemáticas para todas las edades y que motivan y estimulan el aprendizaje activo (Crompton, 2011; Niess, 2005). Además se enfatiza la necesidad de que las matemáticas se estudien en contextos del mundo real para enriquecer la experiencia en el aprendizaje de las matemáticas aplicando los modelos de Aprendizaje Basado en Problemas o Aprendizaje Basado en Investigación (Carrejo y Robertson, 2011; Huang, Liu y Chang, 2012).

Estudios relacionados dan cuenta del tema de la apropiación tecnológica y estrategias de enseñanza, por ejemplo, De la Garza, Alvarado, Glasserman, y Ballesteros (2014), 
describen cómo el uso de recursos educativos abiertos (REA) como estrategia de enseñanza puede incidir en un mejor desempeño en los alumnos de nivel bachillerato. Por su parte, Basilotta y Herrada (2013) realizaron un estudio acerca del aprendizaje a través de proyectos colaborativos con TIC, donde se deja evidente la necesidad de avanzar en las diferentes etapas de apropiación tecnológica. Por último, Segovia, Mérida, González y Olivares (2013) desarrollaron un estudio donde se describe el choque cultural en las aulas, dado la falta de desarrollo de competencias tecnológicas en los profesores.

\section{MÉTODO}

Se siguió una metodología cualitativa con un enfoque de estudio de casos, donde cada caso tiene una particularidad especial (Yin, 2006). En el estudio los casos fueron seleccionados por su singularidad en la docencia de la enseñanza de las matemáticas y se llevó a cabo con una muestra por conveniencia de acuerdo con Merriam (2009), constituida por dos profesoras del nivel primaria, de primer y tercer grado, y sus respectivos grupos con 18 estudiantes cada uno.

Fue importante que, a partir del tema, se determinaran las categorías de la temática a estudiar y los indicadores que permitieron el estudio de las categorías a través de preguntas concretas, para que posteriormente se verificara el cumplimiento del principio de triangulación que garantizara la validez interna de la investigación (Yin, 1994).

Para dar validez a los resultados y partiendo del objetivo de la investigación de analizar el proceso de apropiación tecnológica que se desarrolla en docentes de nivel primaria al utilizar estrategias de enseñanza vinculadas al uso de dispositivos tecnológicos para el reforzamiento de conceptos matemáticos, se tomaron en cuenta tres categorías, comenzando por la categoría de Etapas de apropiación tecnológica conformada por tres indicadores: Acceso, adopción y transformación constituidos a partir del modelo de Carroll et al. (2003). Cada uno de estos indicadores indagó a través de preguntas concretas sobre la etapa de apropiación tecnológica para identificar qué piensa y hace el docente al usar la tecnología y poder ubicarlo en algún nivel de apropiación.

La segunda categoría Estrategias de enseñanza, se conformó de tres indicadores: Estrategias de enseñanza vinculadas a herramientas tecnológicas, tipos de recursos tecnológicos utilizados y utilización de recursos tecnológicos; permitiendo identificar de qué manera los docentes integran estrategias de enseñanza en conjunto con recursos tecnológicos dentro de su docencia.

La tercera y última categoría Construcción de aprendizaje matemático indagó a través de tres indicadores: Conceptualización de números naturales, conceptualización de conceptos multiplicativos y aprendizaje que permitieron conocer de qué manera la utilización de estrategias de enseñanza basadas en tecnología apoyan la enseñanza y reforzamiento de temas de matemáticas. 
Las anteriores fueron las categorías de análisis preconfiguradas; Rossman y Marshall (2010) enuncian que el investigador puede preconfigurar lo que va a investigar antes de la recolección de los datos, generar categorías de datos para recolectar. En la Figura 1 se muestran las categorías e indicadores de esta investigación.

\begin{tabular}{|c|c|}
\hline Categorias & Indicadores \\
\hline \multirow[t]{3}{*}{$\begin{array}{l}\text { 1) Etapas de apropiación tecnológica: } \\
\text { En esta categoria se explican los niveles de } \\
\text { apropiación tecnológica que van adquiriendo } \\
\text { los docentes. }\end{array}$} & $\begin{array}{l}\text { a) Acceso a la tecnologia: } \\
\text { Se visualiza el nivel de aprendizaje de los } \\
\text { recursos tecnologicos que tienen las docentes. }\end{array}$ \\
\hline & $\begin{array}{l}\text { b) Adopción de la tecnologia: } \\
\text { Se visualiza el nivel de dominio de los recursos } \\
\text { tecnológicos que tienen las docentes. }\end{array}$ \\
\hline & $\begin{array}{l}\text { c) Apropiación de la tecnología: } \\
\text { Se visualiza el nivel de aprovechamiento de los } \\
\text { recursos tecnológicos que tienen las docentes. }\end{array}$ \\
\hline \multirow{3}{*}{$\begin{array}{l}\text { 2) Estrategias de enseñanza: } \\
\text { En esta categoría de conceptualizanlas } \\
\text { estrategias de enseñanza vinculadas al uso de } \\
\text { recursos tecnológicos, de las cuales se apoyan } \\
\text { las profesoras en su proceso de apropiación } \\
\text { tecnológica. }\end{array}$} & $\begin{array}{l}\text { a) Estrategias de enseñanza vinculadas a } \\
\text { herramientas tecnológicas: } \\
\text { Se visualizanlas estrategias de enseñanza usa das } \\
\text { porlas docentes y que se vinculan a las TIC. }\end{array}$ \\
\hline & $\begin{array}{l}\text { b) Tipos de recursos tecnológicos utilizados: } \\
\text { Aqui se especifican los recursos tecnológicos } \\
\text { que tienen impacto en su utiliza ción conlas } \\
\text { estrategias de enseñanza. }\end{array}$ \\
\hline & $\begin{array}{l}\text { c) Utilización de recursos tecnológicos: } \\
\text { Se visualiza la forma de integrar tanto } \\
\text { estrategias de enseñanza como recursos } \\
\text { tecnológicos dentro de las clases. }\end{array}$ \\
\hline \multirow[t]{3}{*}{$\begin{array}{l}\text { 3) Construcción de aprendizaje } \\
\text { matemático: } \\
\text { En esta categoria se menciona la forma que se } \\
\text { pueden integrar los recursos tecnológicos en la } \\
\text { enseñanza y reforzamiento de matemáticas. }\end{array}$} & $\begin{array}{l}\text { a) Conceptualización de nimmeros naturales: } \\
\text { Se visualiza el conocimiento del concepto de } \\
\text { numeros naturales que tienen los alumnos de } \\
\text { primer grado. }\end{array}$ \\
\hline & $\begin{array}{l}\text { b) Conceptualización de conceptos } \\
\text { multiplicativos: } \\
\text { Se visualiza el conocimiento del concepto de } \\
\text { multiplicación que tienen los alumnos de tercer } \\
\text { grado. }\end{array}$ \\
\hline & $\begin{array}{l}\text { c) Aprendizaje: } \\
\text { Se considera la influencia que tiene la } \\
\text { utilización de estrategias de enseñanza basadas } \\
\text { en tecnologia para el aprendizaje de conceptos } \\
\text { matemáticos. }\end{array}$ \\
\hline
\end{tabular}

Figura 1. Categorías e indicadores de estudio de la investigación (elaboración propia).

Se utilizaron como instrumentos para la recolección de datos los siguientes: (a) entrevista semi-estructurada aplicada y grabada individualmente a las profesoras, conformada por 20 preguntas para indagar sobre su aprendizaje, dominio y aprovechamiento hacia los recursos tecnológicos dentro de su docencia; sobre las estrategias de enseñanza vinculadas a recursos tecnológicos y sobre la construcción de aprendizaje matemático mediante la utilización de estrategias de enseñanza basadas en tecnología; (b) bitácora de observación de clase para la indagación de todas las categorías y sus indicadores, se registró a detalle el desarrollo de las clases, recursos utilizados y actividades y las actitudes de los docentes y alumnos, y (c) análisis de documentos significativos que consistió en la consulta de diferentes documentos realizados por los alumnos: ejercicios de pretest, postest y ejercicios de clase, los cuales indagaron la categoría construcción de 
aprendizaje matemático y sus indicadores y; de aquellos que sirvieron de guía al docente como planes de clase y documentos de la materia, indagándose las categorías de etapas de apropiación tecnológica, estrategias de enseñanza y construcción de aprendizaje matemático, con la finalidad de importar información adicional y complementaria que permitió describir la investigación con más precisión. 


\section{RESULTADOS}

De acuerdo con la aplicación de los instrumentos se presentan los resultados de la investigación correspondientes a cada categoría y se separan tomando como base a cada profesor y su grupo de estudiantes.

\section{1) Etapas de apropiación tecnológica}

La profesora 1 (primer grado) se dice dispuesta a trabajar con la tecnología aunque menciona que para el uso de las iPads se necesita más tiempo para capacitación. Menciona en la entrevista que sus niveles de aprendizaje, de dominio y de aprovechamiento en el uso de los recursos tecnológicos son los básicos e indispensables pues no tiene tiempo para aprender a manejarlos más y sacar provecho a las herramientas y aplicaciones, se encuentra en la etapa de acceso a la tecnología.

La profesora 2 (tercer grado) menciona en la entrevista que ha alcanzado un nivel básico de conocimiento de las herramientas tecnológicas; pero su creatividad la lleva a trabajar constantemente en la elaboración de nuevos materiales que desarrolla y presenta muy bien al frente del grupo, se encuentra en la etapa de adopción de la tecnología.

\section{2) Estrategias de enseñanza}

La profesora 1 menciona que sus estrategias de enseñanza se han beneficiado con la integración de las TIC pues sus clases se hacen más fáciles, atractivas y digeribles para sus alumnas, además menciona que "el pizarrón interactivo es menos pesado y aburrido que el pizarrón tradicional". No desarrolló un plan de clase para todas las sesiones por lo que las clases se realizaron en muy corto tiempo, se repitieron actividades o bien se perdieron de vista los objetivos y aprendizajes esperados. La profesora utiliza las herramientas tecnológicas para dar repasos y refuerzos de ciertos temas. Mencionó que basa su decisión para el uso de estrategias vinculadas a las TIC en la facilidad que estas brindan a sus clases. Entre las estrategias que utilizó se encuentra el planteamiento de objetivos, el aprendizaje colaborativo y materiales didácticos atractivos; menciona además que desde hace diez años ha usado dentro de su enseñanza las herramientas tecnológicas y quisiera tener más conocimiento y sacarle provecho a todo lo que se pueda hacer con estas. El pizarrón interactivo fue el dispositivo tecnológico con el que más les gustó trabajar a las alumnas (ver Figura 2). 


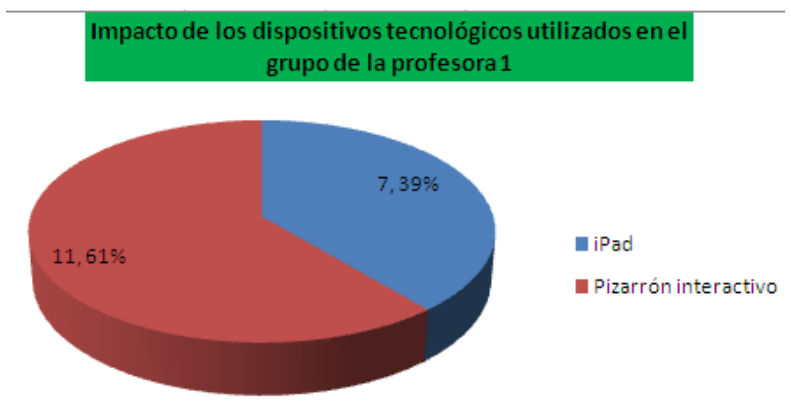

Figura 2. Preferencia de uso de dispositivos tecnológicos utilizados.

La profesora 2 siempre utiliza planes de clase, para ella son muy necesarios ya que muestran las competencias y los aprendizajes esperados, así como la secuencia de la clase y las estrategias de enseñanza aplicables. La profesora trabajó con el pizarrón interactivo y con iPads, además de materiales digitales. Con el apoyo de recursos tecnológicos enriquece sus estrategias de enseñanza tradicionales al sustituir material tangible por material digital lo que a las niñas les resulta mejor. Se pudo observar la utilización de estrategias de enseñanza como: planteamiento de objetivos, mapas conceptuales, ilustraciones, evaluación. Utilizó las TIC con base en tres puntos que menciona en la entrevista: que le sirvan para el aprendizaje esperado, que este al nivel de los alumnos y que se ajusten al tiempo disponible. Utilizó actividades y ejercicios basados en internet y apoyadas con el pizarrón interactivo provocando la emoción de las niñas que externaban expresiones como: jotra vez!, iuna vez más por favor!, se oían aplausos. La utilización del iPad en la clase genera niñas felices, participativas y colaboradoras quienes externan comentarios como: iahora yo!, ¡Miss yo no he pasado!, imuy bien! El iPad fue el dispositivo tecnológico con el que más les gustó trabajar a las alumnas (ver Figura 3).

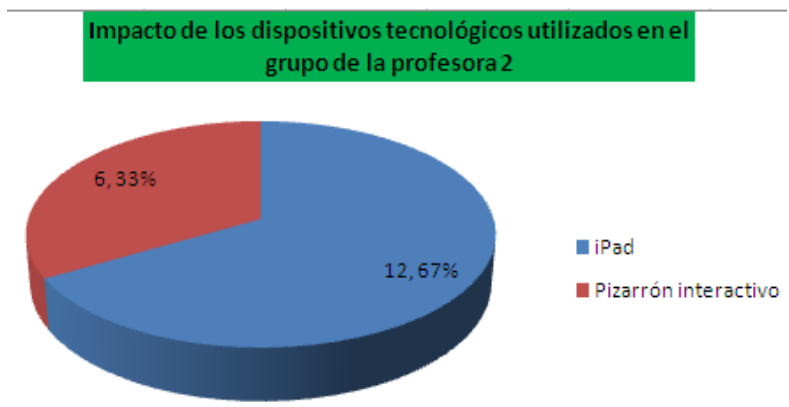

Figura 3. Preferencia de uso de dispositivos tecnológicos utilizados.

\section{3) Construcción de aprendizaje matemático}

Profesora 1. Con los resultados de los ejercicios y cotejando con lo esperado en los planes de clase, se obtuvo que el cumplimiento del aprendizaje esperado si se logró, pero no se lograron las competencias señaladas de resolución de problemas de manera autónoma y valorar procedimientos y resultados. 
Profesora 2. En la entrevista la profesora menciona que con el uso de la tecnología se cumple en gran medida el logro de los objetivos, se desarrollan aprendizajes significativos logrando mejores resultados en las niñas que son distraídas y se agilizan los procesos de enseñanza de esta asignatura. En este sentido, después de la aplicación de los ejercicios de evaluación se obtuvo que la mayoría de las alumnas mejoró notablemente su aprendizaje de los temas estudiados; sin embargo "las niñas que son distraídas" (palabras de la profesora) no mostraron una mejora significativa.

Con base en los resultados presentados anteriormente se realizó un análisis de los mismos llevándonos a la obtención de hallazgos significativos que se enuncian a continuación:

\section{1) Etapas de apropiación tecnológica}

\section{Acceso a la tecnología}

En la actualidad, los profesores están conscientes que deben desarrollar habilidades pedagógicas y tecnológicas que les permitan apropiarse de metodologías de enseñanza para aplicarlas a las nuevas generaciones de alumnos. En este sentido, las docentes del estudio mencionaron que al estar actualizadas en conocimientos de tecnología pueden avanzar al ritmo de los alumnos quienes se motivan más al utilizar recursos tecnológicos innovadores en clase. Estas ideas pueden asociarse como algo inicial en la etapa de acceso a la tecnología tal como lo expone González (2008) quien menciona que es fundamental que el profesor adquiera ciertas habilidades, conocimientos y actitudes que lo capaciten para aplicar estrategias innovadoras y modelos alternos que incluyan la enseñanza por medio de las TIC proporcionando al alumno un rol activo en su proceso de aprendizaje. Esto significa que los docentes conocen la importancia de introducir a su docencia el uso de las TIC y que los primeros acercamientos y uso que se dé a la tecnología les permitirán llegar a apropiarse de ésta.

\section{Adopción de la tecnología}

El desarrollo profesional que permitiría que los profesores mejoren habilidades y estrategias basadas en tecnología en sus aulas, parte de recibir una capacitación previa y/o una actualización constante para que se den los cambios esperados. Comentarios emitidos por las profesoras señalan que sería muy importante y necesaria una capacitación en tecnología, pero no solo en el uso de la herramienta, sino mucho más en su aplicación, señalan además que al final todo recae en la manera de que el maestro tenga el conocimiento y lo aplique para que los niños aprendan mejor. Lo anterior se relaciona con la etapa de adopción pues es donde los profesores decidirán si continúan con el uso de la tecnología, Guzmán y Nussbaum (2009) argumentan que la integración de la tecnología incluye llevar la teoría educativa en la práctica y en la aplicación de resultados para potenciar la enseñanza, mencionan además seis dominios interrelacionados que apoyan los procesos de formación de los docentes. En este sentido los programas de capacitación y actualización que se imparten a docentes deben contemplar la integración de la tecnología mostrando lo que se puede obtener de ellas además de su uso tanto instrumental como pedagógico y didáctico, esto permitirá que los 
docentes se sientan acompañados, que pierdan el miedo a la innovación o utilización de las TIC, que crezca su interés y compromiso para continuar a la etapa de apropiación.

\section{Apropiación de la tecnología}

Una vez que los profesores se introducen y avanzan en la incorporación de las TIC debido a los beneficios que esta les proporciona, se despierta en ellos una actitud de mejora continua que beneficia tanto a ellos como a sus alumnos. La profesora de tercer grado que se encuentra en el nivel adopción mencionó que le gustaría aprovechar al 100\% los recursos tecnológicos y desarrollar un portafolio de material digital. Se pudo observar que la docente aprovecha sus ratos libres en la computadora del salón para enviar tareas o preparar material digital para otras asignaturas. Por su parte, la profesora de primer grado del nivel de acceso comentó que si supiera manejar mejor los recursos tecnológicos los aprovecharía más pero que ha mejorado su destreza en la búsqueda de materiales e información. Relacionado con lo anterior y a la luz de lo que indican Carroll et al. (2003) la apropiación de la tecnología no es una actitud definitiva, pues está sujeta a un refuerzo progresivo que puede ir cambiando la evaluación hacia la tecnología por parte del usuario y conducirlo a una desaprobación. De esta forma podemos mencionar que llegar al nivel de la apropiación tecnológica dependerá de la forma en que los docentes se comprometan en la mejora de su proyecto formativo; por ejemplo, en la asignación de espacios de tiempo para aprender de la tecnología dejando de ser este una limitante o fomentar la investigación y autoestudio.

\section{2) Estrategias de enseñanza}

\section{Estrategias de enseñanza vinculadas a herramientas tecnológicas}

La utilización de estrategias de enseñanza apoyadas con tecnología es útil siempre y cuando su aplicación se enfoque en la asignatura que se quiere apoyar además de que beneficie el desarrollo e impartición de la clase y el desempeño de profesores y alumnos durante y después de esta. Las profesoras externan algunos de los beneficios que han encontrado al apoyarse de la tecnología, entre los que se encuentran ahorro de tiempo y esfuerzo al utilizar pizarrón interactivo en vez de tradicional, generación de clases más atractivas y digeribles para los alumnos, desarrollo de clases menos aburridas y en consecuencia mejor actitud de los alumnos al realizar actividades de acuerdo con su nivel, facilidad en la elaboración de material y su reutilización. Partiendo de lo anterior DíazBarriga y Hernández (2002) comparten cinco aspectos que permiten seleccionar y aplicar las estrategias de acuerdo con el contexto de la clase: 1 ) características de los estudiantes, 2) dominio y contenido curricular, 3) meta a la que se quiere llegar, 4) cuidar proceso de enseñanza y aprendizaje y 5) contexto creado con los alumnos. Para encontrar la utilidad de los recursos tecnológicos en la impartición de una asignatura es necesario basarse en parámetros que permitan decidir porqué, para qué y cómo hacer uso de ellos.

Tipos de recursos tecnológicos utilizados 
La gran variedad de recursos tecnológicos que existen actualmente, ofrecen al docente diversas herramientas para realizar actividades acordes a las características de su modalidad educativa y de su enseñanza en diversas asignaturas lo que beneficia la autogestión de tiempo y preparación de los profesores. Al observar que las profesoras utilizaron diversos dispositivos tecnológicos además de recursos digitales variados, se encontró que la utilización del pizarrón interactivo fue el dispositivo que más frecuentemente se utilizó apoyándose de plantillas interactivas, herramientas web como Youtube, páginas interactivas y documentos de Word. Cabe mencionar que el trabajo con las iPads fue menor por su reciente incorporación y en estas se utilizaron apps y páginas interactivas. Lo anterior se apoya de lo que Gómez y Alemán (2011) presentan como una amplia variedad de modalidades educativas que se apoyan de las TIC y que ofrecen diversas características que se adaptan a las necesidades de cada individuo, estas son $e$ learning, b-learning, u-learning y m-learning. Cada una de estas modalidades ofrecen tanto dispositivos tecnológicos como recursos digitales que benefician a los profesores pues pueden elegir entre un abanico de posibilidades, lo que sin duda les ayudará en el manejo de su tiempo y en su preparación.

\section{Utilización de recursos tecnológicos}

El uso apropiado de los recursos tecnológicos con base en saber que se quiere, además de la constancia en su uso y la capacidad de cada docente, permitirá que estos encuentren sentido didáctico de la tecnología e incorporarla de manera significativa en su enseñanza. Lo anterior se puede verificar en el proceso que cada una de las profesoras ha llevado en su incorporación de la tecnología; ambas tienen en promedio diez años de usar la tecnología pero una de ellas se encuentra temerosa, la falta de tiempo y sus habilidades tecnológicas la limitan en encontrar el sentido didáctico de la tecnología; por su parte la otra profesora trabaja de manera constante en buscar y crear actividades interactivas para sus clases lo que le ha permitido encontrar el sentido didáctico de la tecnología. Al respecto, Carroll et al. (2003) mencionan que evaluar a un docente en su proceso de apropiación tecnológica por un corto período de tiempo es insuficiente ya que se necesita una evaluación más profunda de las competencias que se puedan lograr como resultado de un uso persistente. Se considera importante entonces evaluar la frecuencia y la forma de uso de las TIC en sus actividades cotidianas y en sus procesos formativos; es decir, cómo evalúan, adaptan e integran la tecnología en sus prácticas cotidianas.

\section{3) Construcción de aprendizaje matemático}

\section{Conceptualización de números naturales}

Los profesores deben considerar esencial la elaboración de planes de clase de la asignatura de matemáticas, partir de algo concreto y tangible para tener éxito en su actividad y en el aprendizaje esperado; la planeación debe cubrir mínimamente los aprendizajes establecidos y esperados en el currículo de matemáticas además de las estrategias de enseñanza reforzadas con recursos tecnológicos. En algunas de las sesiones observadas se percibió la ausencia de un plan de clase lo cual generó descontrol en la 
secuencia de la misma, no se marcaron tiempos para las actividades, se perdieron de vista las necesidades de aprendizaje, se improvisaron actividades con los recursos tecnológicos provocando repetición de las mismas y en consecuencia no se lograron las competencias y aprendizajes esperados. Al respecto Mistretta (2005) menciona que la forma de llevar a cabo una metodología para evaluar e integrar la tecnología educativa en las clases de matemáticas parte de la determinación del propósito de los recursos tecnológicos que se utilizaran, el tipo de instrucción posible y finalmente la evaluación de los recursos tecnológicos a diferencia de otras estrategias de enseñanza. Una correcta planeación en donde se indiquen contenidos, aprendizajes esperados, acciones, materiales físicos o recursos tecnológicos a utilizar mejoraran la enseñanza de las matemáticas.

\section{Conceptualización de multiplicación}

La inclusión de actividades creativas como parte de estrategias de enseñanza apoyadas por tecnología y que son aplicadas dentro de un plan de clase, permiten al docente una mejor impartición de clase de matemáticas. En el estudio se pudo observar que la profesora de tercer grado de forma creativa elaboró material para utilizarse en el pizarrón interactivo, presentaciones en Power Point, mapas mentales, entre otros; además en sus clases se apreció la versatilidad y creatividad en la utilización de material digital como dibujos, páginas de Internet interactivas, apps, elaboración de mapas conceptuales, que apoyaron el tema de la multiplicación. Al respecto Yushau et al. (2005) argumentan que la tecnología tiene el potencial para la creación de un entorno propicio para involucrar a los estudiantes en las matemáticas, dota a los profesores las herramientas necesarias para la creatividad de la enseñanza y promueve la creatividad en los alumnos lo que los ayudará a visualizar y apreciar los conceptos matemáticos. El desarrollo de la creatividad en los profesores que se apoyan de la tecnología para la impartición de sus clases, promoverá en ellos la acción de búsqueda o creación de nuevas herramientas, además que les permitirá adaptarse a los métodos educativos en los que se desenvuelva.

\section{Aprendizaje}

Es importante que los profesores se apoyen de un modelo de enseñanza mediado por la tecnología en la impartición de la asignatura de matemáticas para el logro de competencias y aprendizajes significativos en los alumnos. Los resultados de los alumnos muestran que cuando no se tiene una estructura que guie el tema de estudio se logran parcialmente los resultados; en ambos grupos de estudio se lograron los aprendizajes esperados pero no se lograron las competencias señaladas dentro del currículo, siendo estas la resolución de problemas de manera autónoma y la valoración de procedimientos y resultados. Al respecto Huang et al. (2012) y Carrejo y Robertson (2011) han aplicado modelos de enseñanza en sus investigaciones como el Aprendizaje Basado en Problemas o Aprendizaje Basado en Investigación para la impartición de la asignatura de matemáticas, los cuales apoyados por la tecnología han logrado muy buenos resultados estimulando el pensamiento crítico y el aprendizaje colaborativo en los estudiantes. Estos modelos pueden ser aplicables a la asignatura de matemáticas siempre y cuando sean bien 
seleccionados, se conozca bien su técnica y lo que se pretende obtener de estos ya que un solo modelo no es suficiente para obtener todo tipo de metas.

\section{CONCLUSIONES}

De acuerdo con la pregunta principal acerca de cómo se da la apropiación tecnológica de docentes de educación primaria de primer y tercer grado, que utilizan estrategias de enseñanza vinculadas a dispositivos tecnológicos como el pizarrón interactivo y tabletas digitales para el reforzamiento de conceptos matemáticos, se puede enunciar que el nivel 1, o etapa de acceso a la tecnología, ocurre cuando los profesores reconocen su nueva realidad educativa y están conscientes en el desarrollo de habilidades pedagógicas y tecnológicas para aplicarlas a nuevas estrategias de enseñanza. En los casos estudiados, las profesoras tenían conocimientos básicos de computación e iniciaban con el uso de los dispositivos tecnológicos como iPads y pizarrón interactivo, los cuales debían ser utilizados dentro de las aulas apoyándose de estrategias de enseñanza para el reforzamiento de temas de matemáticas. En esta primera etapa las profesoras comenzaron a interactuar con los dispositivos tecnológicos mediante la integración de actividades sencillas y estrategias y de esa forma adquirieron ciertas habilidades que les permitieron ir conociendo la utilidad de la introducción de las TIC y aplicar sus conocimientos de computación. En este sentido, una de las docentes alcanzó este nivel de acceso al comenzar la integración de actividades sencillas dentro de sus clases de matemáticas.

Por su parte, el nivel 2, o etapa de adopción de la tecnología, ocurre cuando los profesores después de conocer o definir su nueva forma de trabajo con apoyo de la tecnología, deciden desarrollar y mejorar su proceso de aprendizaje del uso de las TIC para la enseñanza de sus asignaturas; es decir, deciden a través de sus aptitudes encontrar un sentido didáctico a la integración de la tecnología en sus clases. En esta segunda etapa, las profesoras del estudio continuaron utilizando los dispositivos tecnológicos, buscando o elaborando material digital más innovador, interactivo y creativo que reforzara sus clases de matemáticas; además demandaban capacitación y actualización en el uso de los recursos tecnológicos para mejorar las estrategias de enseñanza. En este sentido una de las profesoras alcanzó este nivel debido a que se sentía atraída por la tecnología lo que le ha permitido mejorar a través de ésta sus estrategias de enseñanza.

En cuanto al nivel 3, o etapa de apropiación de la tecnología, ocurre cuando los profesores están convencidos de que es imprescindible la utilización de la tecnología dentro de su docencia y generan, apoyados de los recursos tecnológicos, materiales digitales muy completos, estrategias de enseñanza competitivas o proyectos con firmes propósitos pedagógicos y didácticos. Esta tercera etapa no fue alcanzada por las profesoras del estudio quizá por el poco tiempo que han usado los dispositivos mencionados, además que no se pudo observar una completa utilización de recursos tecnológicos y digitales, 
aunado a otros factores como la falta de tiempo, de capacitación y de modelos concretos para la enseñanza de las matemáticas que les permitan alcanzar esta etapa.

Los resultados de la investigación permitieron identificar que el impacto que tienen los dispositivos tecnológicos hacia los estudiantes depende directamente de la frecuencia en que se utilizan y la forma o aplicación que se les da en las clases, en este sentido destaca que un uso más novedoso e interactivo genera mayor impacto en las alumnas, quienes muestran una actitud más atenta, colaboradora y participativa en el desarrollo de las clases.

Se encontró también que los profesores no cuentan con la destreza requerida o habilidades necesarias para apropiarse de la tecnología rápidamente, ya que aunque lleven varios años de utilizar una computadora no necesariamente quiere decir que serán capaces de integrar apropiadamente el uso de las herramientas tecnológicas con el currículo.

Este estudio puede servir como referencia para otras investigaciones mediante el cual puedan observar el proceso de apropiación tecnológica de los docentes que se involucran en el uso de estrategias de enseñanza apoyadas por tecnología para la impartición de cualquier asignatura.

\section{REFERENCIAS}

BASILOTTA, V. y HERRADA, G. (2013). Aprendizaje a través de proyectos colaborativos con TIC. Análisis de dos experiencias en el contexto educativo. Edutec, Revista Electrónica de Tecnología Educativa, 44. Disponible en: http://edutec.rediris.es/Revelec2/Revelec44/aprendizaje proyectos colaborativ os TIC experiencias.html

CARREJO, D. Y ROBERTSON, W.(2011).Integrating Mathematical Modeling for Undergraduate Pre-Service Science Education Learning and Instruction in Middle School Classrooms.US-China Education Review, 8(4), 499-509.

CARROLL, J., HOWARD, S., PECK, J. y MURPHY, J. (2003).From Adoption to Use: the process of appropriating a mobile phone. Australasian Journal of Information Systems, 10(2), 38-48. doi:10.3127/ajis.v10i2.151.

CELAYA, R., LOZANO, F., y RAMÍREZ, M. S. (2010). Apropiación tecnológica en profesores que incorporan recursos educativos abiertos en educación media superior. Revista Mexicana de Investigación Educativa, 15(45), 487-513.

CENTRO para la TECNOLOGÍA en el APRENDIZAJE (2007).Prepared for Texas Instruments by the Center for Technology in Learning, SRI International. 
COBO, R. C. (2005).Organización de la información y su impacto en la usabilidad de las tecnologías informativas [Tesis de doctorado no publicada]. Barcelona, España: Universidad Autónoma de Barcelona.

COBO, R. C. (2007, diciembre).Aprendizaje adaptable y Apropiación tecnológica: Reflexiones prospectivas. Ponencia presentada en el 3er encuentro de Autoestudio de las Universidades Públicas Mexicanas "La Universidad en México en el año 2030: imaginando futuros". Ciudad Universitaria, México. Recuperado de http://www.laisumedu.org/DESIN Ibarra/autoestudio3/ponencias/ponencia33.p df

CROMPTON, H. (2011). Mathematics in the Age of Technology: There Is a Place for Technology in the Mathematics Classroom. Journal of the Research Center for Educational Technology (RCET), 7(1), 54-66.

DE LA GARZA, R.C., ALVARADO, C., GLASSERMAN, L.D. y BALLESTEROS, M.A. (2014). Recursos educativos abiertos (REA) en el nivel medio superior: ¿mejoran el aprendizaje? Edutec, Revista Electrónica de Tecnología Educativa, 48. Disponible en: http://edutec.rediris.es/Revelec2/Revelec48/n48 Garza Alvarado Glasserman Ballesteros.html

DÍAZ-BARRIGA, F. y HERNÁNDEZ, G. (2002). Estrategias docentes para un aprendizaje significativo. Distrito Federal, México: McGraw-Hill.

GLASSERMAN, L. D. (2013). Aprendizaje activo en ambientes enriquecidos con tecnología. (Disertación doctoral). Tecnológico de Monterrey. Monterrey, Nuevo León, México. Disponible en formato abierto en: http://catedra.ruv.itesm.mx/handle/987654321/743

GÓMEZ, M. G. y ALEMÁN, L. (2011). Administración de proyectos basados en tecnología [eBook]. Monterrey, Nuevo León, México: Editorial Digital del Tecnológico de Monterrey.

GONZÁLEZ, J.C. (2008). TIC y la transformación de la práctica educativa en el contexto de las sociedades del conocimiento. RUSC. Revista de Universidad y Sociedad del Conocimiento, 5(2), 1-8.

GUZMAN, M. A. y NUSSBAUM, M. (2009).Teaching Competencies for Technology Integration in the Classroom. Journal of Computer Assisted Learning, 25, 453-469.

HUANG, T.H., LIU, Y.C. y CHANG, H.C. (2012). Learning Achievement in Solving WordBased Mathematical Questions through a Computer-Assisted Learning System. Educational Technology \& Society, 15(1), 248-259.

MERRIAM, S. (2009).Qualitative research: A guide to design and implementation. San Francisco, California, Estados Unidos: Jossey-Bass. 
MISTRETTA, R. M. (2005). Integrating technology into the mathematics classroom: the role of teacher preparation programs. The Mathematics Educator, 15(1), 18-24.

NIESS, M. L. (2005). Preparing teachers to teach science and mathematics with technology: Developing a technology pedagogical content knowledge. Teaching and Teacher Education, 21(5), 509-523.

RAMÍREZ, M. S. (2009). Recursos tecnológicos para el aprendizaje móvil (mlearning) y su relación con los ambientes de educación a distancia: implementaciones e investigaciones. Revista de investigación educativa, 12 (2), 57-82.

ROSSMAN, G. y MARSHALL, C. (2010). Designing qualitive research. EUA: Congress cataloging-in-Publication Data.

SEGOVIA, B., MÉRIDA, R., GONZÁLEZ, E. y OLIVARES, M.A. (2013). Choque cultural en las aulas: profesores analógicos vs alumnado digital. El caso de Ana. Edutec, Revista Electrónica de Tecnología Educativa, 43. Disponible en:http://edutec.rediris.es/Revelec2/Revelec43/choque cultural aulas profesores a nalogicos alumnado digital.html

YIN, R. K. (1994).Case study research: Design and methods. California, Estados Unidos: Sage.

YIN, R. K. (2006). Case Study Methods. En J.L. Green, G. Camilli, P. B. Elmore (Eds.), Handbook of Complementary Methods in Education Research. Estados Unidos: Routledge.

YUSHAU, B., MJI, A. y WESSELS, D. (2005). The role of technology in fostering creativity in the teaching and learning of mathematics. Pythagoras (62), 12-22.

\section{Para citar este artículo:}

Escobar, J. V.; Glasserman, L. D. \& Ramírez, M. S. (2015). Apropiación tecnológica con pizarrón interactivo y tabletas digitales en profesores de educación básica. EDUTEC, Revista Electrónica de Tecnología Educativa, 53. Recuperado el dd/mm/aa de http://www.edutec.es/revista 